\title{
Evaluation of formulation variables on azithromycin nanoparticles prepared by emulsification solvent diffusion method using quality by design approach
}

\author{
Nguyen-Thach Tung*, Canh-Hung Nguyen, Huu-Manh Nguyen \\ Department of Pharmaceutics, Hanoi University of Pharmacy, Vietnam
}

\begin{abstract}
The study aims to investigate the effect of formulation variables on the characteristics of azithromycin (AZI) nanoparticles using a quality by design approach. AZI nanoparticles were prepared by the emulsification solvent diffusion method. Two critical factors, the ratio of AZI: Eudragit EPO $\left(\mathrm{X}_{1}\right)$ and volume of outer aqueous medium $\left(\mathrm{X}_{2}\right)$, were chosen as independent variables for central composite design. The optimized formulation was further characterized by Fourier transform infrared spectroscopy, X-ray diffractometry, transmission electron microscopy, and dissolution test. The obtained results showed variability of mean particle size, entrapment efficacy, and zeta potential from 200 to $1232 \mathrm{~nm}, 10.78$ to $75.9 \%$, and 31 to $43 \mathrm{mV}$, respectively. The main coefficients indicated that the ratio of AZI: polymer $\left(\mathrm{X}_{1}\right)$ possessed a synergistic effect on mean particle size $\left(\mathrm{Y}_{1}\right)$, and volume of outer aqueous medium $\left(\mathrm{X}_{2}\right)$ had an antagonistic effect on particle size. The interaction between the ratio of AZI: Eudragit EPO $\left(\mathrm{X}_{1}\right)$ and volume of outer aqueous medium $\left(\mathrm{X}_{2}\right)$ exhibited a significant antagonistic effect on entrapment efficacy $\left(\mathrm{Y}_{2}\right)(p<0.05)$. AZI existed in an amorphous state in nanoparticles that were spherical and homogeneous in shape. The nanoparticles revealed the Korsmeyer-Peppas release model, from which AZI was released faster compared to raw material.
\end{abstract}

Keywords:

Azithromycin, Nanoparticles, Emulsification solvent diffusion, Quality-by-design

\section{INTRODUCTION}

Nanoparticles are defined as solid colloidal particles that include both nanospheres and nanocapsules ${ }^{1}$. Many studies have proved the superiorities of nanoparticulated systems including solubility enhancement ${ }^{2}$, better drug release control $^{3}$, higher intracellular uptake than other particulate systems ${ }^{4}$, more effective drug targeting ${ }^{5}$, and improved drug stability ${ }^{6}$. To prepare nanoparticles, there are six classical methods: nanoprecipitation, emulsion-diffusion, double emulsification, emulsion-coacervation, polymer-coating, and layer-by-layer ${ }^{1}$. Among the above methods, the solvent diffusion emulsification method has many advantages in terms of size control, high repeatability between batches, and convenience in scaling ${ }^{7}$. This method was usually used to prepare polymeric nanoparticles using different polymers such as poly (lactic-co-glycolic acid), polycaprolactone, cellulose derivatives, or Eudragit derivatives as polymeric carriers ${ }^{8-}$ ${ }^{10}$. Different researchers ${ }^{10-12}$ studied the effect of process and formulation factors on the properties of nanoparticles prepared by the above methods using conventional experimental designs. However, these designs have demonstrated certain disadvantages, in which changing a single input factor while keeping others constant leads to more experiments than feasible and eliminates the interaction of factors ${ }^{13-14}$.

In such context, quality-by-design (QbD) has been promoted by the United States Food and Drug Administration (USFDA) as a systematic approach to enhance pharmaceutical development through design efforts ${ }^{15}$. The QbD has two main objectives: (a) to design a process in a way that pharmaceutical manufacture consistently meets critical quality attributes, and (b) to

*Corresponding author:

*Nguyen-Thach Tung Email: nguyenthachtung@hup.edu.vn 
understand and control the impact of formulation components and process parameters on the critical quality attributes ${ }^{14}$. To grasp both the main and interaction effects of formulation and process factors, some designs of the experiment (DOE) have been employed. In this particular research, the central composite design was chosen as our DOE, because it can handle many independent variables simultaneously and allows for better estimation in terms of an order than other designs of experiment ${ }^{16}$.

Considering the advantages of QbD, azithromycin (AZI), a broad-spectrum antibiotic against Grampositive and Gram-negative bacteria, was selected as the model drug to prepare nanoparticles. The low bioavailability of azithromycin due to its insolubility in water ${ }^{17}$ would be greatly improved by the use of nanoparticulated systems thanks to their unique physicochemical properties such as ultra-small and controllable size, large surface area to mass ratio, high interactions with microorganisms, and host cells, and structural/functional versatility 18-19. In this study, nanoparticles containing AZI were prepared by the emulsification solvent diffusion method which is effective in controlling the mean particle size and polydispersity index $(\mathrm{PDI})^{1}$. Besides, Eudragit EPO was used as a model polymeric carrier because of the following reasons. First, this polymer EPO has a low glass transition temperature $\left(45^{\circ} \mathrm{C}\right)$ thus allowing for flexibility in preparing nanoparticles ${ }^{20}$. Second, this polymer is insoluble in distilled water but completely soluble in dissolution medium $(\mathrm{pH} 4.5)^{21}$, therefore it might limit the drug leakage from nanoparticles in the diffusion step and easily dissolve in gastric medium to release the drug. Moreover, the price of this polymer is much cheaper than PLGA, one of the most popular polymeric carriers, while having almost similar physical properties. Therefore, it is expected that the knowledge obtained from this polymer can be used to conduct further studies with PLGA.

In short, the main objective of this study was to develop azithromycin nanoparticle formulations using the $\mathrm{QbD}$ approach to understand the influence of two formulation factors, i.e. the ratio of AZI: polymer and volume of the outer aqueous medium, on critical quality attributes of azithromycin nanoparticles (mean particle size, entrapment efficacy, and zeta potential).

\section{MATERIALS AND METHODS}

\subsection{Materials}

Azithromycin (AZI) was obtained from Hebei Dongfeng Pharmaceutical Co., Ltd (China). Eudragit EPO (Eud EPO) was supported by Evonik Co., LTD (Germany). Ethyl acetate and polyvinyl alcohol (Mw 9,000-10,000, $80 \%$ hydrolyzed) were purchased from Sigma-Aldrich (U.S.A). HPLC-grade methanol was purchased from J.T.
Baker (U.S.A). Water was purified by reverse osmosis and was filtered in-house. All other reagents were of analytical grade commercial products and purchased from Beijing Chemical Reagent Factory (China).

\subsection{Preparation of azithromycin nanoparticles}

AZI nanoparticles were prepared by emulsification solvent diffusion method which was referred from previous study ${ }^{22}$. Firstly, AZI and Eudragit EPO were simultaneously dissolved in ethyl acetate to obtain an organic phase. A solution of polyvinyl alcohol (PVA) $0.5 \%$ saturated by ethyl acetate was used as an aqueous phase. The organic phase was slowly injected into the water phase by a syringe fitted with an $18 \mathrm{G}$ needle while stirring by the magnetic bar to obtain oil in the water emulsion. It was emulsified either by probe sonicator (Satorius, Germany, Model LabsonicM) or by probe type homogenizer (CAT scientific, U.S.A, Model Unidriver $\mathrm{X} 1000)$. This emulsion was then added into the outer aqueous medium while gently mixing by a magnetic bar for 20 hours at room temperature. The nanoparticles were harvested by centrifugation at 15,000 rpm (DJB Labcare, Germany, Universal 320R) for 30 minutes. The obtained nanoparticles were washed twice with $10 \mathrm{ml}$ of water. Effect of some process variables (sonication amplitude or homogenization cycles) and formulation variables (volume of ethyl acetate and concentration of PVA) on the particle size of nanoparticles were screened before conducting the design of experiment.

\subsection{Design of experiment}

The central composite design was used to investigate the main, interaction, and quadratic effects of the formulation variables on the properties of azithromycin nanoparticles. In this design, two independent variables were the ratio of AZI in total weight $(1.5 \mathrm{~g})$ of solid materials (AZI and Eud EPO) $\left(\mathrm{X}_{1}, \%\right)$ and volume of outer aqueous medium $\left(\mathrm{X}_{2}, \mathrm{ml}\right)$, in which the three levels (low, medium, and high level) of $X_{1}$ were 10, 25, and $40 \%$, respectively. $X_{2}$ was set at 200,300 , and $400 \mathrm{ml}$, respectively. Particle size $\left(\mathrm{Y}_{1}\right)$, encapsulation efficacy $\left(\mathrm{Y}_{2}\right)$, and zeta potential $\left(\mathrm{Y}_{3}\right)$ were chosen as dependent variables. MODDE 8.0 software (Umetrics, Sweden, Version 8.0) was used for the design, analysis, and plotting of the response surface plot and its contour plot.

\subsection{Drug entrapment efficiency}

The obtained AZI nanoparticles were dried in a vacuum drying oven (Daihan Labtech, Korea, Model LVO-2040) at $40^{\circ} \mathrm{C}$ for $24 \mathrm{~h}$. The dried nanoparticles were dissolved in $5 \mathrm{ml}$ ethanol, sonicated for $5 \mathrm{~min}$, and supplemented to $100 \mathrm{~mL}$ phosphate buffer saline, $\mathrm{pH}$ 6.0. The samples were filtered through $0.45 \mu \mathrm{m}$ (Satorius, 
Germany, Model Minisart RC 25) and analyzed using an HPLC method following guidelines in the Vietnamese Pharmacopoeia Version 4.0. The HPLC system consisted of an isocratic pump (Agilent, U.S.A., Model G1311C), a manual injector (Agilent, U.S.A., Model G1328C), a column thermostat (Agilent, U.S.A., Model G1316A), and a multi-wavelength detector (Agilent, U.S.A., Model G1315D). Detector output was integrated and digitalized using the Agilent ChemStation software (Agilent, U.S.A., Model 1200 Series HPLC system). The column used was a C18 (Zorbax SB, 4.6× $150 \mathrm{~mm}, 5 \mu \mathrm{m}$ particle size, Agilent, U.S.A.). The detector was set at $215 \mathrm{~nm}$. The mobile phase containing methanol: distilled water: concentrated ammonia solution at a ratio of 80:19.9:0.1 was delivered at $1 \mathrm{~mL} / \mathrm{min}$ at room temperature. The injection volume was $100 \mu \mathrm{L}$ and the total run time for a sample was about $10 \mathrm{~min}$.

The entrapment efficiency (\%EE) was calculated from the equation:

$\mathrm{EE}(\%)=\underline{\text { Mass of drug in dried nanopartides }} \times 100$

Mass of drug used in formulation

\subsection{Determination of physical properties}

Particle size distribution and zeta potential analysis of the AZI nanoparticles was carried out by photon correlation spectroscopy (PCS) with the help of Malvern Zetasizer (Malvern Instruments, UK, Model ZetasizerNano ZS90). Samples were appropriately diluted with ultra-purified water. Measurements were taken at $25^{\circ} \mathrm{C}$, and each value was measured in triplicate.

The crystallinity of AZI, Eudragit EPO, and nanoparticles was evaluated with an X-ray diffractometer (Siemens, Germany, Model D500) with $\mathrm{Cu}$-Kal radiation and Ni filter. X-ray diffraction data were collected at room temperature in the range of $10^{\circ}<2 \theta<50^{\circ}$. Before measurement, nanoparticles were lyophilized using a freeze dryer (Labconco, U.S.A, Model740030).

FT-IR spectra were obtained on a Fourier transform infrared spectroscopy (Bruker Optics, Germany, Model IFS-66/S) using the potassium bromide $(\mathrm{KBr})$ disk method. One to two mg of sample was mixed with $150 \mathrm{mg}$ of spectra-grade $\mathrm{KBr}$ and pressed into a disk of $12 \mathrm{~mm}$ diameter using carver hydraulic press (Carver, U.S.A., Model 3912). Samples were analyzed from 600$4000 \mathrm{~cm}^{-1}$ with an instrument resolution of $0.1 \mathrm{~cm}^{-1}$.

The morphology and structure of the AZI nanoparticles were studied using transmission electron microscopy (TEM) (Jeol, Japan, Model JEM-1010). The sample was diluted with distilled water at a ratio of 1:100. A drop of the sample obtained after dilution was placed on copper grids. Any excess liquid was drawn off with filter paper. The grid surface was then air-dried at room temperature and observed in a transmission electron microscope at 40-100 kV.

\subsection{Determination of in-vitro drug release}

The nanoparticles were also evaluated for AZI release by dialysis membrane diffusion technique developed by Zhang, et al. ${ }^{23}$. The AZI nanoparticles equivalent to $12.5 \mathrm{mg}$ of the drug were suspended in $5 \mathrm{ml}$ of water and added into a dialysis bag (Spectrum ${ }^{\circledR}$ Laboratory, U.S.A, Membrane MWCO 12000-14000 Daltons). This bag was soaked in $250 \mathrm{ml}$ phosphate buffer saline $(\mathrm{pH}$ 4.5 ) at $37^{\circ} \mathrm{C} \pm 0.5^{\circ} \mathrm{C}$ under $100 \mathrm{rpm}$ stirring. The dissolution rate of AZI from samples into the medium was measured using the dissolution apparatus type 2 (Erweka, Germany, Model DT 600). Five milliliters of aliquot were withdrawn at predetermined time intervals of 15 , 30, 60, and 180 minutes and filtered through $0.45 \mu \mathrm{m}$ membranes (Satorius, Germany, Model Minisart RC 25). The medium was replaced with $5 \mathrm{ml}$ of fresh buffer each time. Withdrawn samples were analyzed using a UV spectrophotometer (Hitachi, Japan, Model U-1800) at $482 \mathrm{~nm}$. The sulfuric acid was used as the colordeveloping agent.

\subsection{Data analysis}

The data was calculated using Excel (Microsoft., USA). MODDE 8.0 software (Umetrics, Sweden, Version 8.0) was used for the design, analysis, and plotting of the response surface plot and its contour plot. The dissolution profile of the observed and predicted data was compared using a similar factor $\left(\mathrm{f}_{2}\right)$, which is described by the following equation ${ }^{24}$ :

$$
\mathrm{f}_{2}=50 \times \log \left\{\left[1+\frac{1}{\mathrm{n}} \sum_{\mathrm{t}=1}^{\mathrm{n}}\left(\mathrm{R}_{\mathrm{t}}-\mathrm{T}_{\mathrm{t}}\right)^{2}\right]^{-0.5} \times 100\right\}
$$

where $\mathrm{n}$ represents the number of sampling points. $T_{i}$ and $R_{i}$ represent the percentage of drug dissolution rate of the observed and predicted samples at each sampling point (i).

\section{RESULTS AND DISCUSSION}

\subsection{Screening of process and formulation variables}

\subsubsection{Effect of process variables}

Nanoparticles were prepared by the emulsification solvent diffusion method. Before conducting the design of the experiment, the effect of some main factors on particles size and PDI of the produced nanoparticles were determined. For the dispersion apparatus, probe sonicator and rotor-stator homogenizer were used (Figure 1). Generally, it was easy to obtain nanoparticles of 200 $\mathrm{nm}$ and PDI under 0.2 when using a probe sonicator. Meanwhile, as the homogenization cycles of the homogenizer increased from 3,900 to $9,800 \mathrm{rpm}$, the particle size and PDI were still over $300 \mathrm{~nm}$ and 0.2 , respectively. It could be explained that the homogenizer created more 


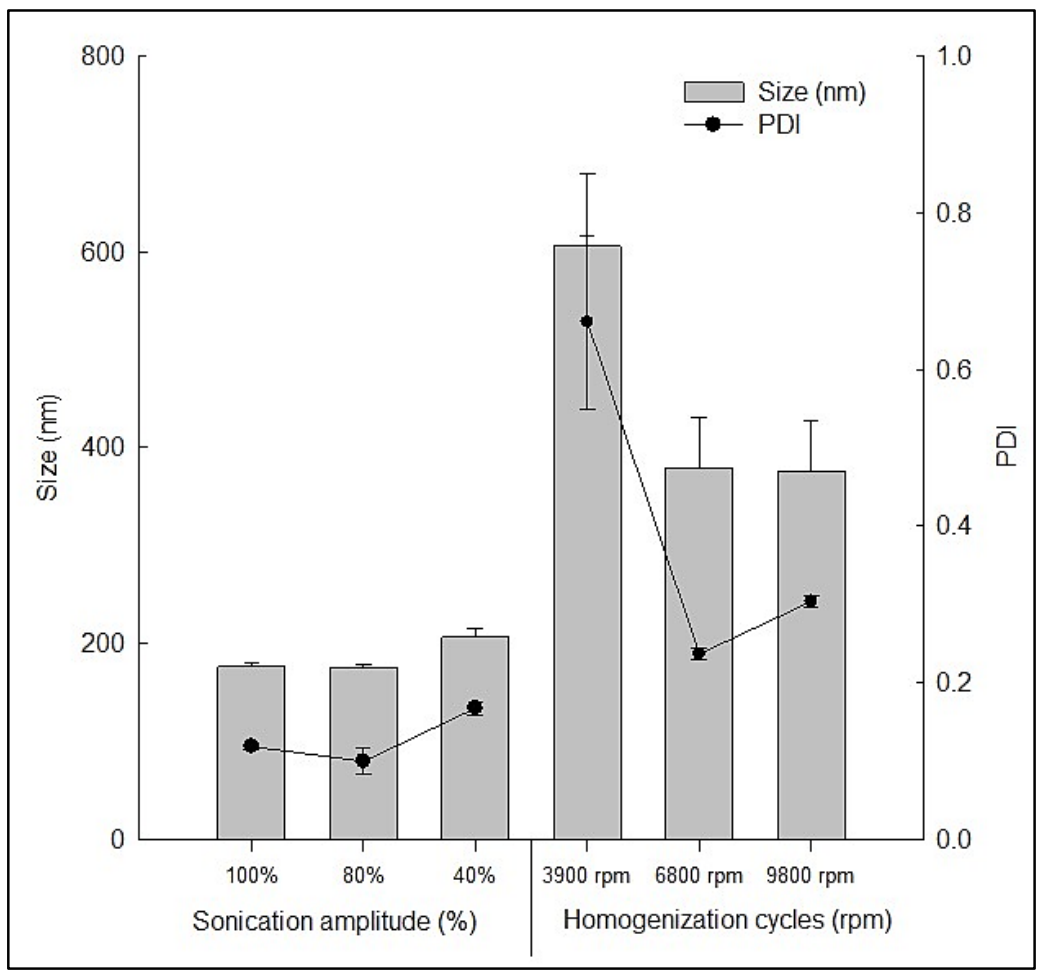

Figure 1. Effect of sonication amplitude (\%) and homogenization cycle (rpm) on particle size and PDI of nanoparticles (n=3, Mean \pm S.D).

Table 1. Effect of sonication time on particle size and PDI of nanoparticles ( $n=3$, Mean \pm S.D).

\begin{tabular}{llcc}
\hline Sonication time (minute) & Sample & Size (nm) & PDI \\
\hline 12 minutes & Blank nanoparticles & $176 \pm 3$ & $0.12 \pm 0.01$ \\
& AZI loaded nanoparticles & $200 \pm 18$ & $0.14 \pm 0.20$ \\
\hline 30 minutes & Blank nanoparticles & $271 \pm 22$ & $0.17 \pm 0.02$ \\
& AZI loaded nanoparticles & $1092 \pm 112$ & 1.00 \\
\hline
\end{tabular}

bubbles than the probe sonicator, thus preventing the contact between the two phases. Besides, the high turbulence of the organic phase in the water phase caused by the homogenizer made the diffusion and evaporation process of ethyl acetate run out of control, leading to PDI that was always higher than expected. In contrast, the gentle dispersion with sonication duty cycle around $0.7 \mathrm{~s}$ helped the oil drops stable in the water phase thereby avoiding unexpected solvent diffusion and evaporation. When using the ultrasonic machine in combination with a magnetic stirrer, the size of nanoparticles was under $200 \mathrm{~nm}$ and the PDI was close to 0.1. Due to its many advantages over high-speed homogenizers (convenience, low energy consumption, effective nanoparticle size control), the ultrasonic machine with $100 \%$ sonication amplitude was chosen to conduct the next studies.

When the sonication time increased from 12 to 30 minutes, the particle size of blank nanoparticles almost doubled while that of AZI-loaded nanoparticles increased by 5 times (Table 1). It was due to the clumping of the organic phase when the sonication time was prolonged. The high temperature caused by the long sonication time promoted evaporation of ethyl acetate, thus forming polydispersity solid particles right in the emulsification stage ${ }^{25}$. The role of the diffusion step in controlling particle size was blurred. Therefore, the sonication time was fixed at 12 minutes with the next samples. This kind of result was slightly different from the result in graphene nanofluids in a previous review study by Madderla Sandhya ${ }^{26}$, who reported that after reaching the optimum time, there is no change in particle size. This difference might be explained by the properties of the polymeric carrier (Eudragit EPO) versus graphene and the preparation methods of emulsification solvent diffusion versus precipitation. Besides, results in Table 1 also showed that the size of samples containing AZI increased from 1.13 to 4.02 times compared to the blank nanoparticles. Assumingly, both azithromycin (pKa 8.5) and Eudragit EPO (pKa 10.0) had the weakly basic property; therefore, they had the positive charge in distilled water. Consequently, when increasing the amount of AZI, the repulsion phenomenon of AZI and Eud EPO possibly caused the leak of drug out of drug-loaded nanoparticles to form the polydisperse colloids.

\subsubsection{Effect of formulation variables}

Besides the effect of process factors, some 


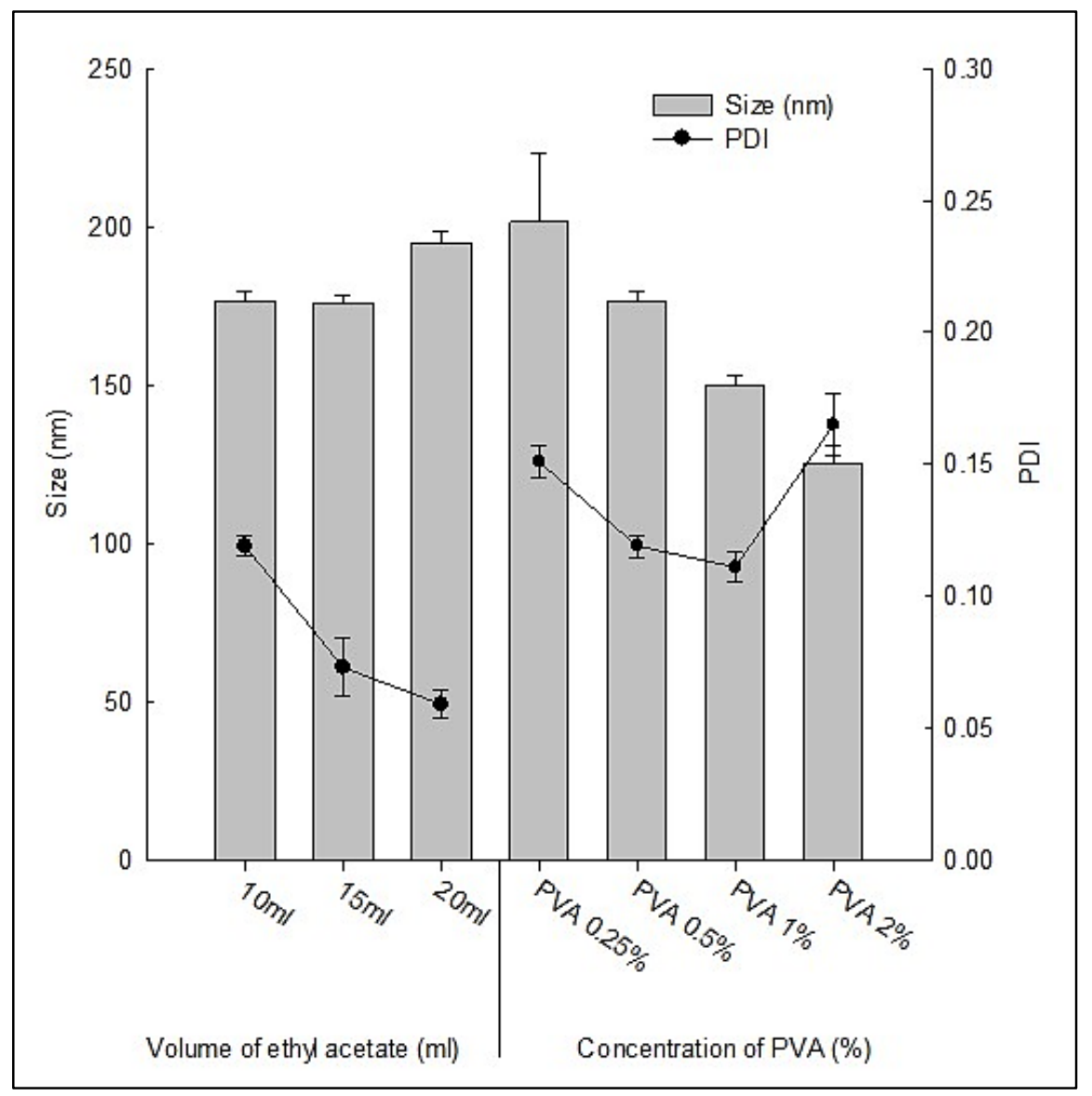

Figure 2. Effect of volume of the organic phase (ethyl acetate, ml) and concentration of PVA (\%) in water phase on particle size and PDI of nanoparticles (Mean \pm S.D).

Table 2. Independent variables measured responses of the central composite design ( $n=3$, Mean \pm S.D).

\begin{tabular}{|c|c|c|c|c|c|}
\hline $\begin{array}{c}\text { Exp } \\
\text { No }\end{array}$ & $\begin{array}{c}\mathbf{X}_{\mathbf{1}} \\
(\text { Ratio of AZI, \%) } \\
{[-1(10 \%) ; 0(25 \%) ; 1(40 \%)]}\end{array}$ & $\begin{array}{c}\mathbf{X}_{\mathbf{2}} \\
(\text { Volume of outer aqueous medium, ml) } \\
{[-1(200 \mathrm{ml}) ; 0(300 \mathrm{ml}) ; 1(400 \mathrm{ml})]}\end{array}$ & $\begin{array}{c}\mathbf{Y}_{\mathbf{1}}(\mathbf{n m}) \\
\text { (Particle size) } \\
\quad(\mathrm{n}=3)\end{array}$ & $\begin{array}{c}\mathbf{Y}_{\mathbf{2}}(\boldsymbol{\%}) \\
\text { (Encapsulation efficacy) } \\
(\mathrm{n}=1)\end{array}$ & $\begin{array}{c}\mathbf{Y}_{\mathbf{3}}(\mathbf{m V}) \\
\text { (Zeta potential) } \\
(\mathrm{n}=3)\end{array}$ \\
\hline 1 & -1 & -1 & $243 \pm 2$ & 10.78 & $+35.9 \pm 1.7$ \\
\hline 2 & 1 & -1 & $1232 \pm 86$ & 71.00 & $+31.0 \pm 6.9$ \\
\hline 3 & -1 & 1 & $200 \pm 3$ & 75.90 & $+34.0 \pm 1.8$ \\
\hline 4 & 1 & 1 & $201 \pm 1$ & 19.60 & $+33.9 \pm 11.4$ \\
\hline 5 & -1 & 0 & $229 \pm 4$ & 46.93 & $+35.6 \pm 2.2$ \\
\hline 6 & 1 & 0 & $220 \pm 1$ & 59.00 & $+39.3 \pm 2.7$ \\
\hline 7 & 0 & -1 & $244 \pm 1$ & 33.81 & $+43.0 \pm 7.5$ \\
\hline 8 & 0 & 1 & $248 \pm 3$ & 35.70 & $+37.6 \pm 1.4$ \\
\hline 9 & 0 & 0 & $222 \pm 2$ & 11.20 & $+37.9 \pm 2.9$ \\
\hline
\end{tabular}

formulation variables including volume of ethyl acetate (organic phase) and concentration of PVA in water phase were also investigated. To make it easier for the screening step, the blank nanoparticles were prepared with the basic compositions including $1.5 \mathrm{~g}$ Eudragit EPO in ethyl acetate as organic phase, $50 \mathrm{ml}$ PVA $0.5 \%$ saturated by $5 \mathrm{ml}$ ethyl acetate as aqueous phase, and $400 \mathrm{ml}$ PVA $0.1 \%$ as the diluted phase. The volume of ethyl acetate would affect the viscosity and micromixing of the organic phase in the aqueous phase. According to Sébastien, et al. ${ }^{27}$, micromixing, i.e. the mixing of two phases at a small scale, is governed by viscous-convective deformation of fluid elements and is followed by molecular diffusion. Ethyl acetate, an organic solvent with a viscosity of $426 \mu \mathrm{Pa} \cdot \mathrm{s}(0.426 \mathrm{cP})$ at $25^{\circ} \mathrm{C}$, was used in this study as the organic phase to dissolve the drug and also saturated in the aqueous phase. Therefore, the different amounts of this solvent might create the different viscosity of the organic phase and affect the molecular diffusion. The role of the organic solvent fraction to the nanoparticle size was also confirmed in the result of a systematic study conducted by HernándezGiottonini $^{8}$ on the effects of the primary formulation parameters involved in the preparation of PLGA nanoparticles, via the emulsification technique.

On the other hand, a high amount of ethyl 
acetate also increased the volume of the diffusion phase and solvent evaporation time. Consequently, the purpose of this step was to determine a proper volume of ethyl acetate to adequately dissolve $1.5 \mathrm{~g}$ of solid components while maintaining a particle size of around $200 \mathrm{~nm}$. Figure 2 showed that even when the volume of EA was 10,15 , or $20 \mathrm{ml}$, the mean particle sizes and PDI were all within the accepted range, i.e., 176.7;175.9;195 nm and $0.119 ; 0.073 ; 0.059$, respectively. However, $10 \mathrm{ml}$ EA was selected as the organic phase for subsequent experiments because of the shortest evaporation time compared to the other screened volumes of EA.

In terms of the impact of the aqueous phase, the effect of concentrations of PVA on size and PDI were determined. As shown in Figure 2, the higher the concentration of PVA was used, the lower the particle size was obtained. Besides, as the amount of PVA increased from 0.25 to $1 \%$ causing a reduction in the free energy, the PDI of AZI nanoparticles decreased from 0.15 to 0.11 . According to the study of Pineda-Reyes ${ }^{28}$, PVA concentration $(0.2-1 \%)$ can stabilize globules in the emulsion and hinder aggregation during diffusion in the solid-liquid interface, thus avoiding fusion and the formation of agglomerates and effective, nonionic surfactant emulsifiers that reduce the size of nanoparticles. However, due to the Osward-ripening phenomena, PDI was increased to 0.17 when the concentration of PVA was $2 \%$. PVA is also known as a surfactant, therefore, it also can enhance drug solubility and lead to the formation of supersaturated areas of the drug which then agglomerate to create polydispersible nanoparticles. Consequently, $1 \%$ PVA was selected for the design of experiment step.

\subsection{Design of experiment}

Based on results obtained from the screening step, all of the screened process and formulation parameters were fixed for the design of experiment. As the independent variables, the ratio of AZI $\left(\mathrm{X}_{1}\right)$ and volume of the outer aqueous phase $\left(X_{2}\right)$ were selected. To evaluate the main and interaction effect of the two input variables on particle size $\left(\mathrm{Y}_{1}\right)$, entrapment efficacy $\left(\mathrm{Y}_{2}\right)$, and zeta potential $\left(\mathrm{Y}_{3}\right)$, the $\mathrm{QbD}$ approach was used. Multiple linear regression analysis, response surface plots, and their contour plots were used to evaluate these data. The value of coefficients reflected the effect of independent variables $\mathrm{X}_{1}, \mathrm{X}_{2}$, and their interaction $\left(\mathrm{X}_{1} * \mathrm{X}_{2}\right)$ on the dependent variables. A positive coefficient indicated a synergistic effect; meanwhile, a negative one reflected an antagonistic effect ${ }^{16}$. The impact of individual coefficients was determined by ANOVA test using the software MODDE 8.0. In this case, each dependent variable (Y) was described by the following quadratic equation:

$$
\mathrm{Y}=\mathrm{b}_{0}+\mathrm{b}_{1} \mathrm{X}_{1}+\mathrm{b}_{2} \mathrm{X}_{2}+\mathrm{b}_{3} \mathrm{X}_{1} \mathrm{X}_{2}+\mathrm{b}_{4} \mathrm{X}_{1}^{2}+\mathrm{b}_{5} \mathrm{X}_{2}^{2}
$$

Where, $b_{0}$ was the intercept and $b_{1}-b_{5}$ were coefficients for the factors $X_{1}, X_{2}$, and their interaction. The value of dependent variables $\left(Y_{1}, Y_{2}\right.$, and $\left.Y_{3}\right)$ and the regression coefficients of independent variables were shown in Tables 2 and Table 3, respectively. These data were used to investigate the impact of input variables on particle size, entrapment efficacy, and zeta potential.

\subsubsection{Influence of input variables on particle size (Y1)}

The mean particle size of the nanoparticles obtained was from 200 to $1232 \mathrm{~nm}$ depending on the levels of independent variables. Regarding the main factors, Table 3 and Figure 3 showed that when the ratio of AZI increased, the nanoparticle size was dependent on the volume of the outer aqueous medium. Specifically, at a high volume of outer aqueous medium and high ratio of AZI, the nanoparticle size was small, and entrapment efficiency was low. Meanwhile, at a low volume of outer aqueous medium and high ratio of AZI, there was an increase in both the nanoparticle size and entrapment efficiency. This meant that the volume of the outer aqueous medium partly controlled the size of nanoparticles. Specifically, at the low volume of the outer aqueous medium, AZI nanoparticles were aggregated to form large nanoparticles size. Moreover, at the high volume of the outer aqueous medium, AZI could leak out of emulsion to the outer medium and losing in the washing steps used to harvest nanoparticles.

The response surface and its contour plot also illustrated the synergistic influence of this main factor on particle size (Figure 4). In contrast, the minus value of $b_{2}(-178.27)$ indicated that the volume of the outer aqueous medium had an antagonistic effect on particle size. It was attributed to the fact that the higher volume of the outer aqueous medium accelerated the diffusion of the internal phase into the external phase to quickly form monodispersity nanoparticles and avoid the agglomeration of droplets. Rahman, et al. also found that the reduction of volume of external phase increased viscosity of organic phase that impacted the shearing efficiency of the stirrer. This phenomenon accelerated the aggregation of semi-solid particles thus increasing mean particle size $^{13}$.

Besides the impact of main factors, the interaction effect of the ratio of AZI with the volume of outer aqueous on mean particle size was also evaluated and shown in Figure 3a. The plot displayed the change of mean particle size in the response when the ratio of AZI varied from 10 to $40 \%$, and the volume of the outer aqueous medium was set at both low $(200 \mathrm{ml})$ and high levels $(400 \mathrm{ml})$. Theoretically, when the two lines were parallel there was no interaction between these two factors. When the two lines crossed each other, there was a strong interaction ${ }^{29-30}$. Consequently, as indicated in Figure $3 \mathrm{a}$, the mean particle size was strongly influenced 

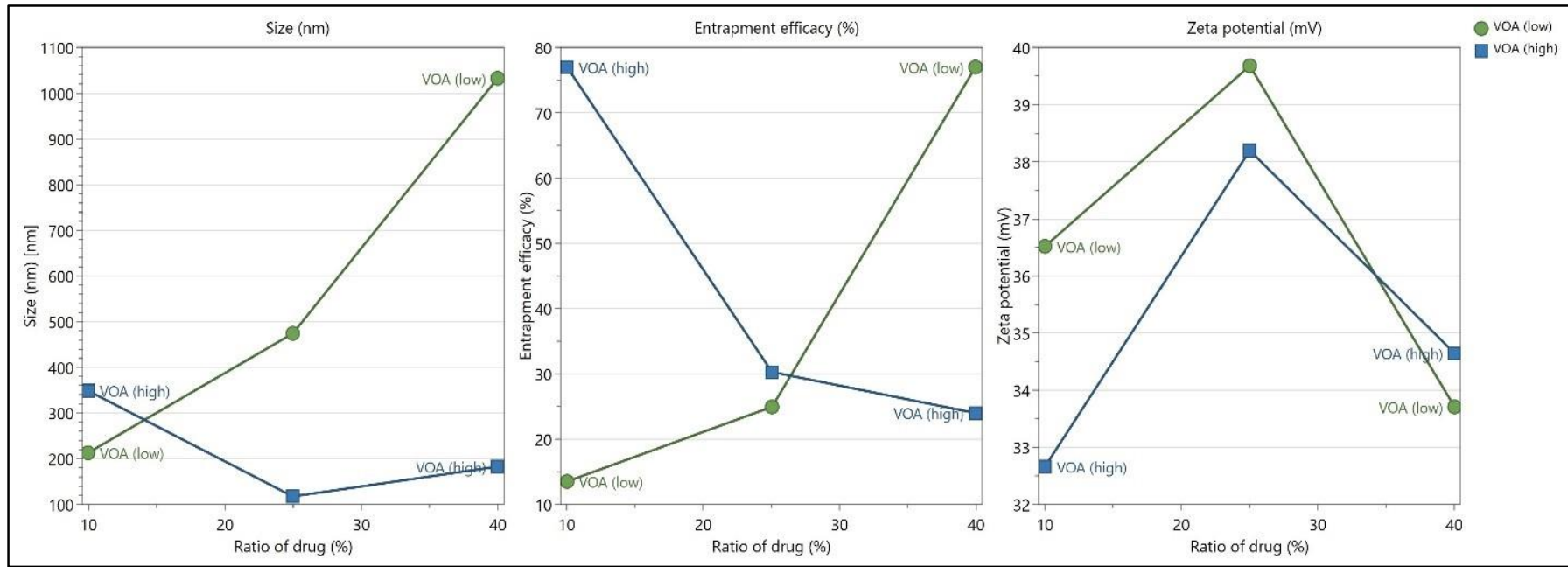

Figure 3. Interaction effect of volume of outer aqueous (VOA, ml) and the ratio of AZI (\%) on a) size (nm) b) entrapment efficacy (\%EE), and c) zeta potential $(\mathrm{mV})$ of azithromycin nanoparticles.

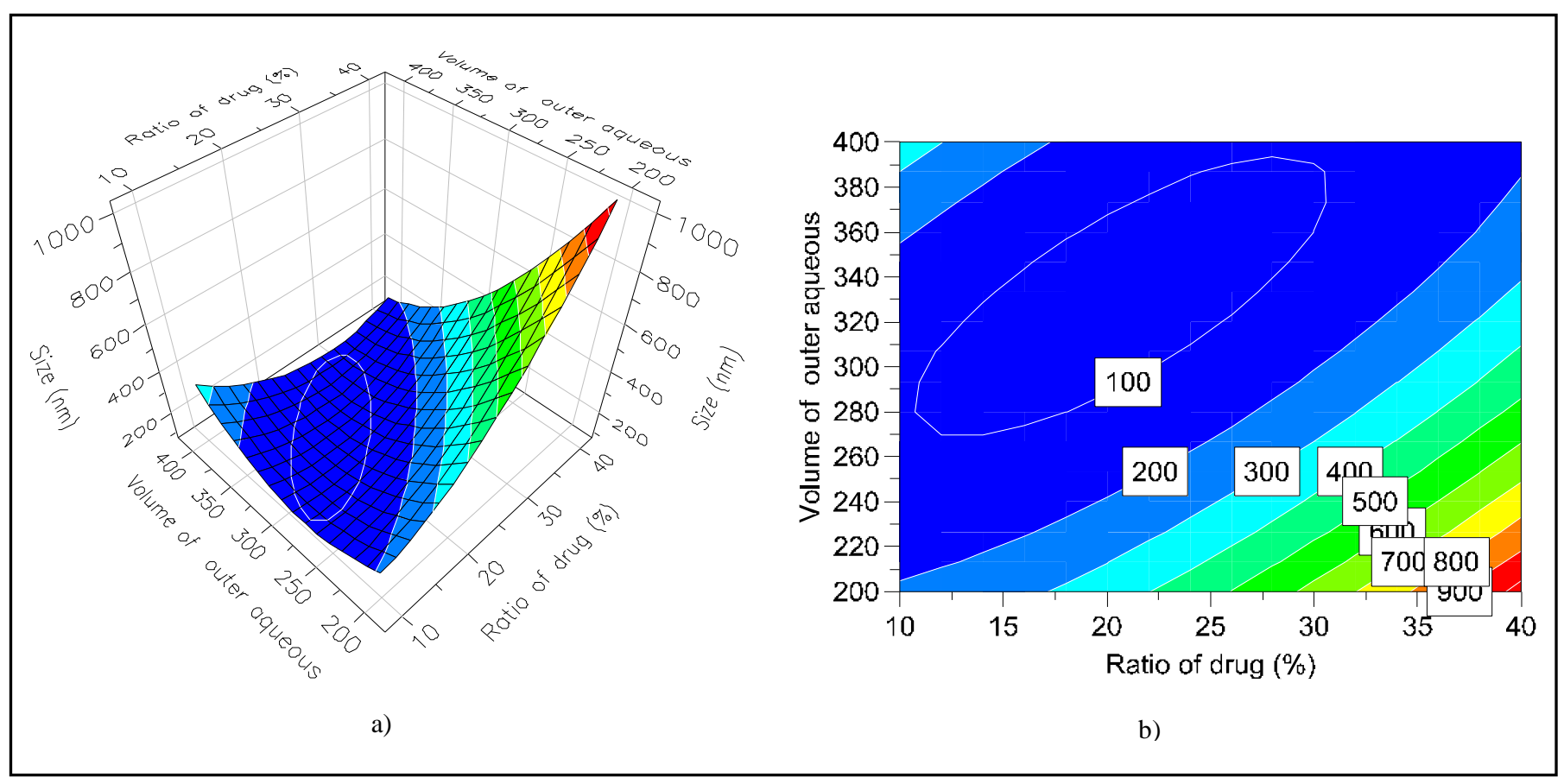

Figure 4. Effect of the volume of outer aqueous and ratio of AZI on particle size using a) response surface plot and b) its contour plot.

Table 3. Regression results of the measured responses.

\begin{tabular}{|c|c|c|c|c|c|c|}
\hline & \multicolumn{2}{|c|}{$Y_{1}(\mathrm{~nm})$} & \multicolumn{2}{|c|}{$Y_{2}(\%)$} & \multicolumn{2}{|c|}{$\mathrm{Y}_{3}(\mathrm{mV})$} \\
\hline & Coefficient & $\mathbf{p}^{*}$ & Coefficient & $\mathbf{p}^{*}$ & Coefficient & $\mathbf{p}^{*}$ \\
\hline Constant & 123.89 & 0.57 & 25.51 & 0.08 & 40.67 & 0.00 \\
\hline $\mathbf{X}_{1}$ & 163.37 & 0.22 & 2.67 & 0.65 & -0.21 & 0.90 \\
\hline $\mathbf{X}_{2}$ & -178.27 & 0.19 & 2.60 & 0.66 & -0.73 & 0.66 \\
\hline $\mathbf{X}_{1} * \mathbf{X}_{1}$ & 149.67 & 0.48 & 20.30 & 0.11 & -4.56 & 0.18 \\
\hline $\mathbf{X}_{2} * \mathbf{X}_{2}$ & 171.17 & 0.42 & 2.09 & 0.83 & -1.73 & 0.56 \\
\hline \multirow[t]{2}{*}{$\mathbf{X}_{1} * \mathbf{X}_{2}$} & -247.05 & 0.15 & -29.13 & 0.02 & 1.20 & 0.56 \\
\hline & $\mathrm{R}^{2}=0.77$ & & $\mathrm{R}^{2}=0.90$ & & $\mathrm{R}^{2}=0.58$ & \\
\hline
\end{tabular}

*The level of statistical significance 
by the interaction between the ratio of AZI and the volume of the outer aqueous medium. Given the fact that the $p$-value was higher than 0.05 , which was regarded as statistical insignificance, the minus value of coefficient $b_{3}(-247.05)$ in Table 3 did demonstrate an antagonistic effect on particle size as a result of the interaction between these two input variables. In addition, the positive value of the quadratic coefficients $\left(\mathrm{X}_{1} \mathrm{X}_{1}\right.$ and $\left.\mathrm{X}_{2} \mathrm{X}_{2}\right)$ denoted a synergistic influence on mean particle size.

\subsubsection{Influence of input variables on entrapment efficacy} (Y2)

The entrapment efficacy of AZI in nanoparticles ranged from 10.78 to $75.9 \%$. The fact that the $\mathrm{R}^{2}$ value was 0.90 for entrapment efficacy indicated a significant fit to the tested model. Taking into consideration the main effects, results in Table 3 underlined that both independent variables, i.e., the ratio of AZI $\left(\mathrm{X}_{1}\right)$ and volume of outer aqueous medium $\left(\mathrm{X}_{2}\right)$, had a synergistic effect on entrapment efficacy. The high drug concentration $\left(\mathrm{X}_{1}\right)$ in the formulation brought about more AZI available for entrapment. Similarly, Figure $3 b$ showed that the samples with a lower ratio of the drug $(10 \%)\left(\mathrm{X}_{1}\right)$ and higher volume $(400 \mathrm{ml})$ of outer aqueous medium $\left(X_{2}\right)$ had a high entrapment efficiency. This could be explained by the following reasons. First, the large volume of the outer medium made it easy for ethyl acetate to diffuse, and solid particles were formed rapidly. The process of diffusion from a solid to liquid medium will be more difficult than liquid-liquid diffusion to reduce drug $\operatorname{loss}^{22}$. Second, the low ratio of the drug in total weight of solid material $(1.5 \mathrm{~g})$ meant that the higher polymer ratio (Eud EPO) was used and increased the viscosity of the emulsion droplet, thereby reducing the diffusion coefficient of the drug. When the solid particles have formed, the high polymer ratio also increased the diffusion distance of the drug molecules in the particles to the environment, which declined drug loss (Figure 5).

However, this finding was different from the study conducted by Rahman, et al. ${ }^{13}$. In their study, cyclosporine A-PLGA nanoparticles were prepared using the emulsification-solvent evaporation technique, and the result indicated that the volume of the external phase had an adverse effect on the entrapment. This was explained by the fact that more volume was available for the drug to diffuse from the internal to external phase, which also decreased the viscosity of the system and further increased the diffusion of the drug. The difference between the two studies may result from different mechanisms to form nanoparticles: emulsificationsolvent evaporation technique versus emulsificationsolvent diffusion technique.

However, regarding the interaction effect of the ratio of AZI and volume of outer aqueous on entrapment efficacy (\%EE), the two lines crossing each other in
Figure $3 \mathrm{~b}$ meant a strong interaction between these two factors on entrapment efficacy. Furthermore, as shown by the $p$-value in Table 3 , the interaction of $\mathrm{X}_{1} * \mathrm{X}_{2}$ on entrapment efficacy was statistically significant $(p<0.05)$. Besides, the minus value of coefficient $b_{3}(-29.13)$ in Table 3 exhibited the antagonistic effect on entrapment efficacy of this interaction. This phenomenon suggested that the simultaneous increase of volume of outer aqueous medium and ratio of drug: polymer decreased the drug entrapment efficacy and vice versa. As illustrated in the response surface and its contour plot (Figure 5), the highest level of the drug entrapment efficacy (75.9\%) was obtained when the volume of diffusion medium and the ratio of AZI was set at $400 \mathrm{ml}$ of the outer aqueous medium and $10 \%$ of drug ratio. Additionally, the positive value of the quadratic factors $\left(X_{1} X_{1}\right.$ and $\left.X_{2} X_{2}\right)$ in Table 3 indicated the synergistic influence of these variables on drug entrapment efficacy (\%).

\subsubsection{Influence of input variables on zeta potential (Y3)}

Zeta potential is the charge on the surface of particles in water, which acts as an indication of the physical stability of nanoparticles dispersion. Generally, the value of $\pm 30 \mathrm{mV}$ assures the stability of dispersed systems ${ }^{13}$. The zeta potential of the studied nanoparticles between 31 and $43 \mathrm{mV}$ proved the physical stability of the screened systems.

Given the main and quadratic factors, regression coefficients in Table 3 showed that the ratio of AZI $\left(\mathrm{X}_{1}\right)$, volume of outer aqueous medium $\left(\mathrm{X}_{2}\right), \mathrm{X}_{1} \mathrm{X}_{1}$, and $\mathrm{X}_{2} \mathrm{X}_{2}$ had an antagonistic effect on particle size. However, the ANOVA test clarified that these regression coefficients were not considered to be statistically significant ( $p>0.05$ ) (Table 3). The Zeta potential of the blank nanoparticles was $+44.2 \mathrm{mV}$. And this value was generally reduced when entrapped by azithromycin (Figure 6). Similar results were observed in previous studies, Rahman, et al. used the Plackett-Burman design to understand the effect of dependent variables on the quality of protein-loaded PLGA nanoparticles ${ }^{13}$. In their study, they also concluded that none of the evaluated factors was significant in predicting the value of zeta potential, which suggested that this dependent value was very hard to control and predict.

Regarding the interaction effect of the ratio of AZI and volume of outer aqueous on zeta potential, the regression coefficients in Table 3 indicated their synergistic effect on zeta potential. The interaction plot (Figure 3c) showed the two trendlines. When the ratio of the drug increased from low (10\%) to medium (25\%) level, the two lines were almost parallel and there was no interaction between the two input variables. However, when the ratio of AZI increased from medium (25\%) to high level (40\%), the two lines crossed, and there was a strong interaction. 


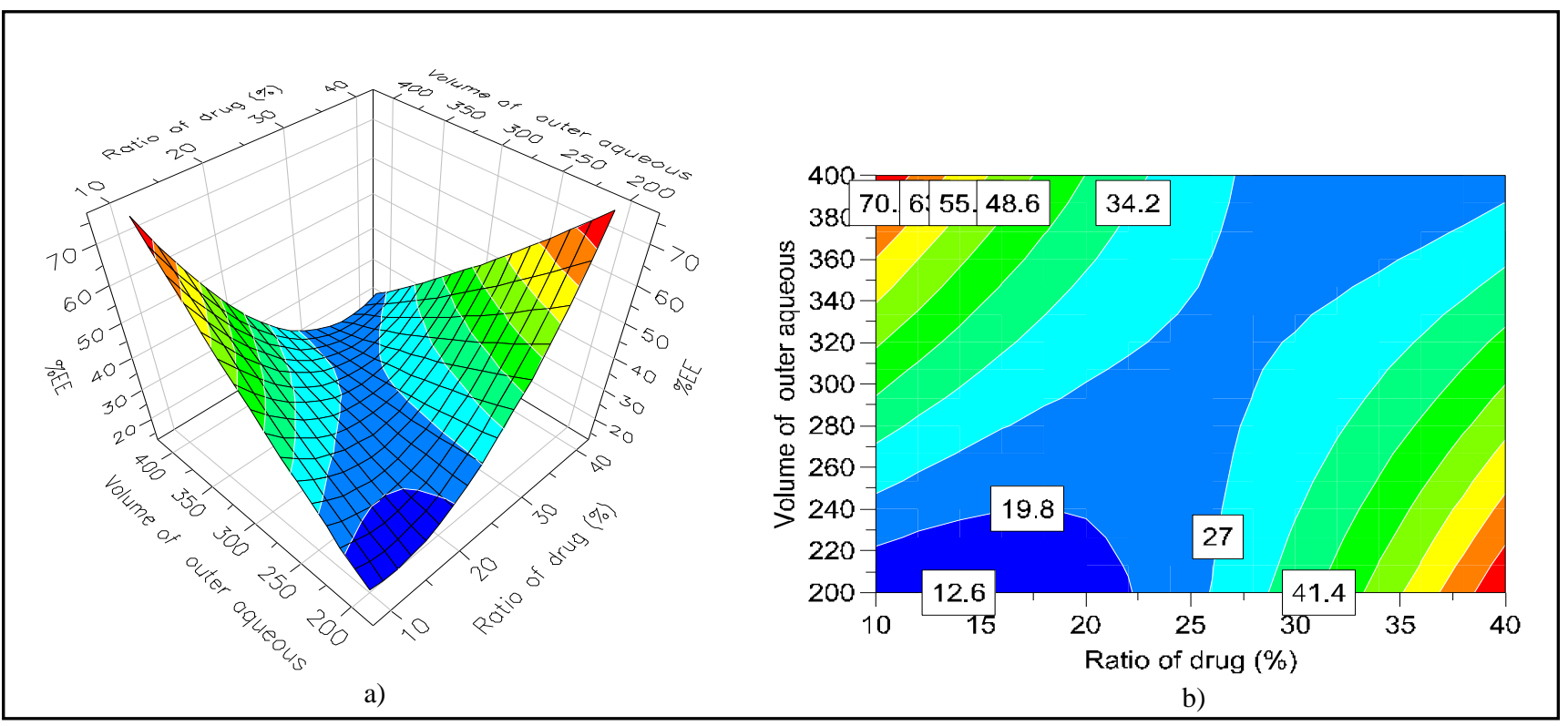

Figure 5. Effect of the volume of outer aqueous and ratio of AZI on entrapment efficacy using a) response surface plot and b) its contour plot.

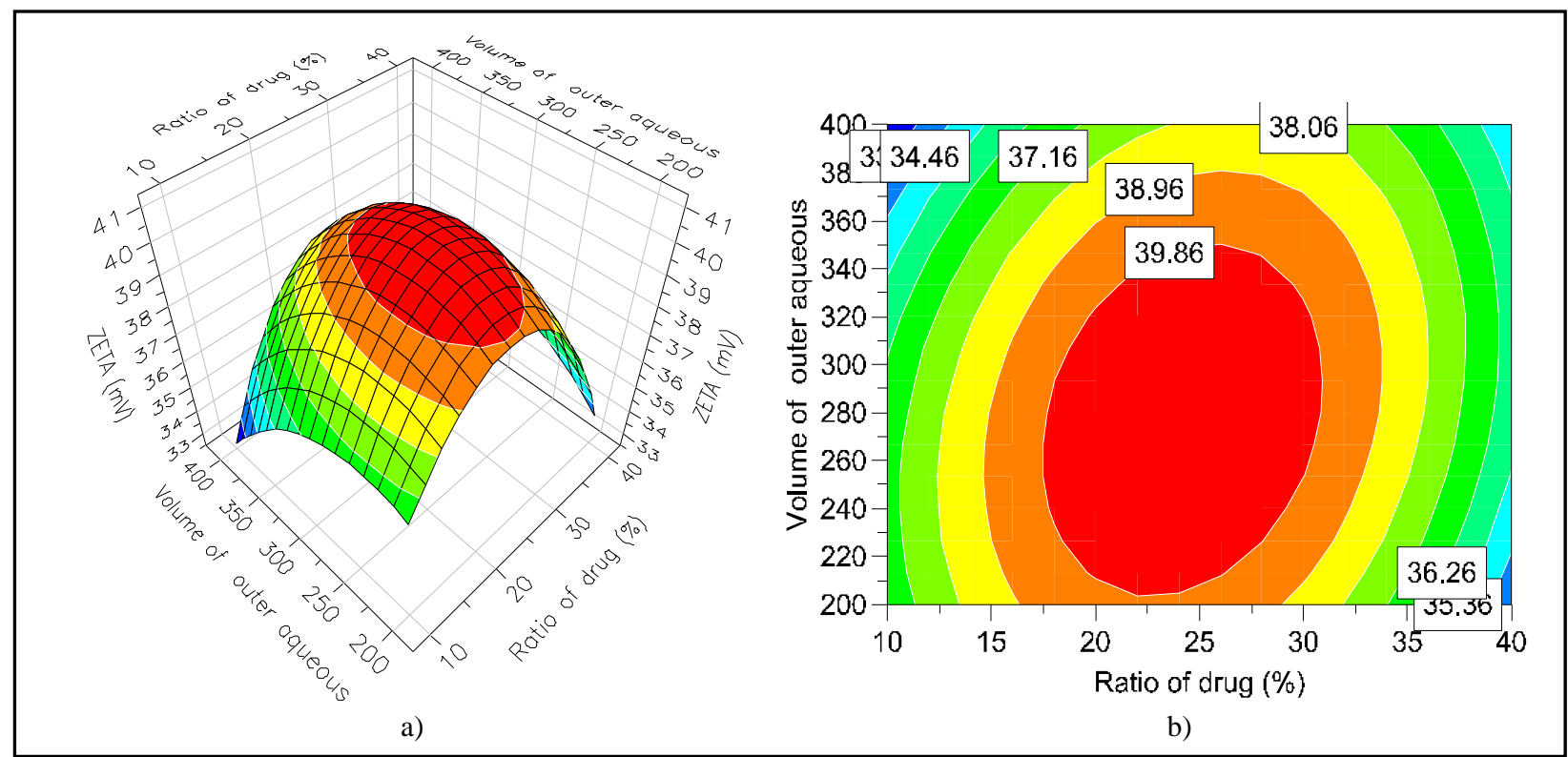

Figure 6. Effect of the volume of outer aqueous and ratio of AZI on zeta potential using a) response surface plot and b) its contour plot.

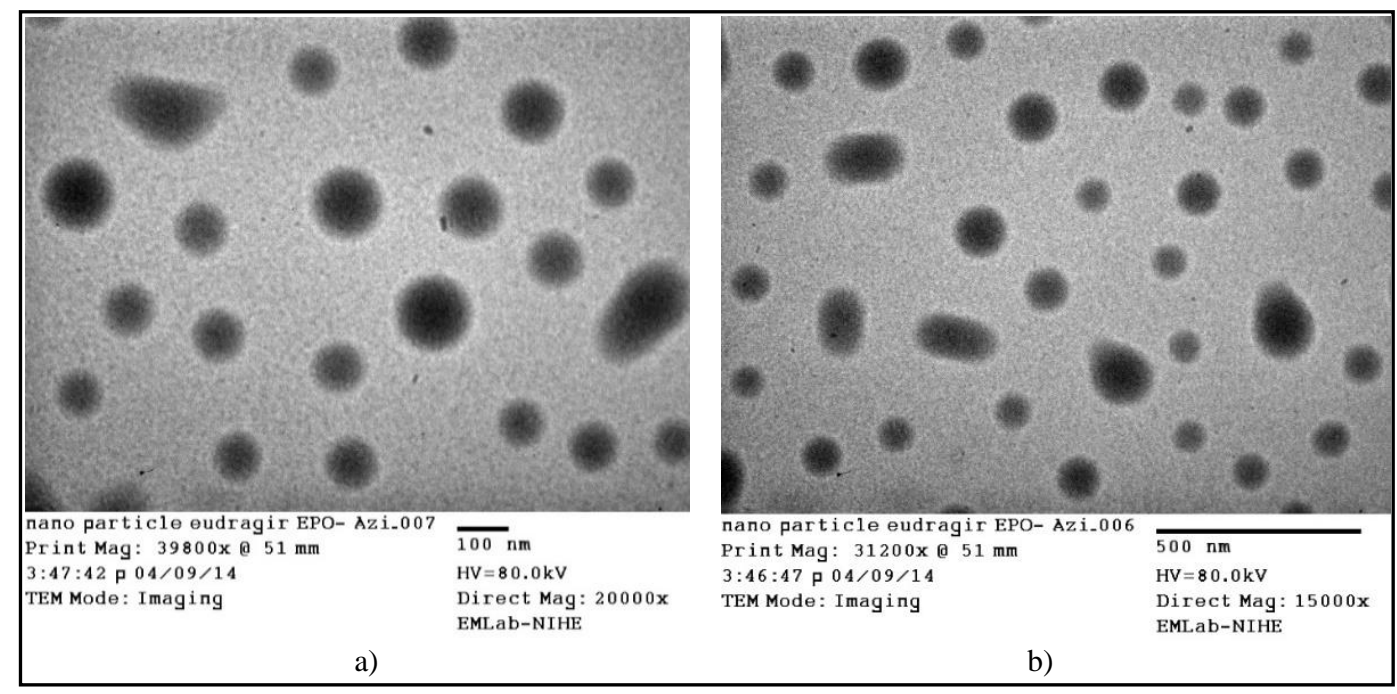

Figure 7. TEM images of azithromycin nanoparticles at the scale bars of a) $100 \mathrm{~nm}$ and b) $500 \mathrm{~nm}$. 


\subsection{Characterization of azithromycin nanoparticles}

To determine the physicochemical properties of nanoparticles, TEM, X-ray, and FTIR analyses were carried out. The photon correlation spectroscopy method was employed to determine the mean particle size of nanoparticles; however, the morphology of nanoparticles could not be observed. TEM results showed that the majority of nanoparticles had a rough shape, and the particles size was under $200 \mathrm{~nm}$ with the limited size distribution (Figure 7). These descriptions agreed with those obtained by using the photon correlation method.

Besides, an understanding of the physical state of azithromycin in nanoparticles was gained by studying powder X-ray diffractograms of AZI, Eudragit EPO, and AZI-loaded nanoparticles. Generally, the intense sharp peaks that appeared in the diffraction pattern of AZI at a $2 \theta$ value of 10 to $30^{\circ}$ indicated the crystalline state in AZI raw material (Figure 8). However, these specific peaks almost disappeared in X-ray diffraction of AZIloaded nanoparticles. It can, therefore, be concluded that azithromycin transformed from crystalline state to amorphous state while encapsulating into the nanoparticles $^{31}$.

Further evaluation of the interaction between azithromycin and Eudragit EPO was carried out using the FTIR experiment. Eudragit EPO had seven H-bond acceptors specified by carbonyl groups $(-\mathrm{C}=\mathrm{O})$ at 1729 $\mathrm{cm}^{-1}$. Meanwhile, AZI had $5 \mathrm{H}$-bond donors $(-\mathrm{OH})$ indicated by a peak at $3494 \mathrm{~cm}^{-1}$ and 14 acceptors groups. FT-IR spectroscopy of AZI-loaded nanoparticles showed a slight movement of a hydroxyl group from 3494 to
$3048 \mathrm{~cm}^{-1}$ (Figure 9) ${ }^{32}$. This meant that AZI and Eudragit EPO might form a hydrogen bond.

To evaluate the dissolution model of AZI from nanoparticles, the dissolution test was modified from a previous study by Zhang, et al. ${ }^{33}$. The $\mathrm{pH} 4.5$ was used for the release study after some careful considerations. First, Azithromycin has been known as a weakly basic drug thus having higher solubility in an acidic medium. However, according to our previous study ${ }^{34}$, this drug was found unstable in very low $\mathrm{pH}$ medium such as $\mathrm{pH}$ $1-3$, thus $\mathrm{pH}$ between 4 and 5 would be considered. At the same time, Eudragit EPO is completely soluble in an acidic dissolution medium $(\mathrm{pH} 4.5)$ and is not soluble at pH over 5-6. Also, $\mathrm{pH} 4.5$ has also been one of the general dissolution mediums usually used in bioequivalence studies.

With X-ray analysis, it was confirmed that AZI encapsulated into nanoparticles and was in the amorphous state, which led to a faster release rate. Besides, the nano size of particles was also attributed to the higher dissolution rate of AZI than raw material (Figure 10). To get an insight into the mechanism of drug release from nanoparticles, the release data were fitted into zero, first order, Korsmeyer-Peppas, HicksonCrowell, and Higuchi, and the determination coefficient values were $0.95,0.23,0.99,0.57$, and -1.22 , respectively. The obtained results showed that the AZI release pattern was best fitted in the Korsmeyer-Peppas model. The drug release mechanism can be figured out from the Korsmeyer-Peppas equation:

$$
\underline{\mathrm{M}}_{\mathrm{t}}=\mathrm{Kt}^{\mathrm{n}}
$$

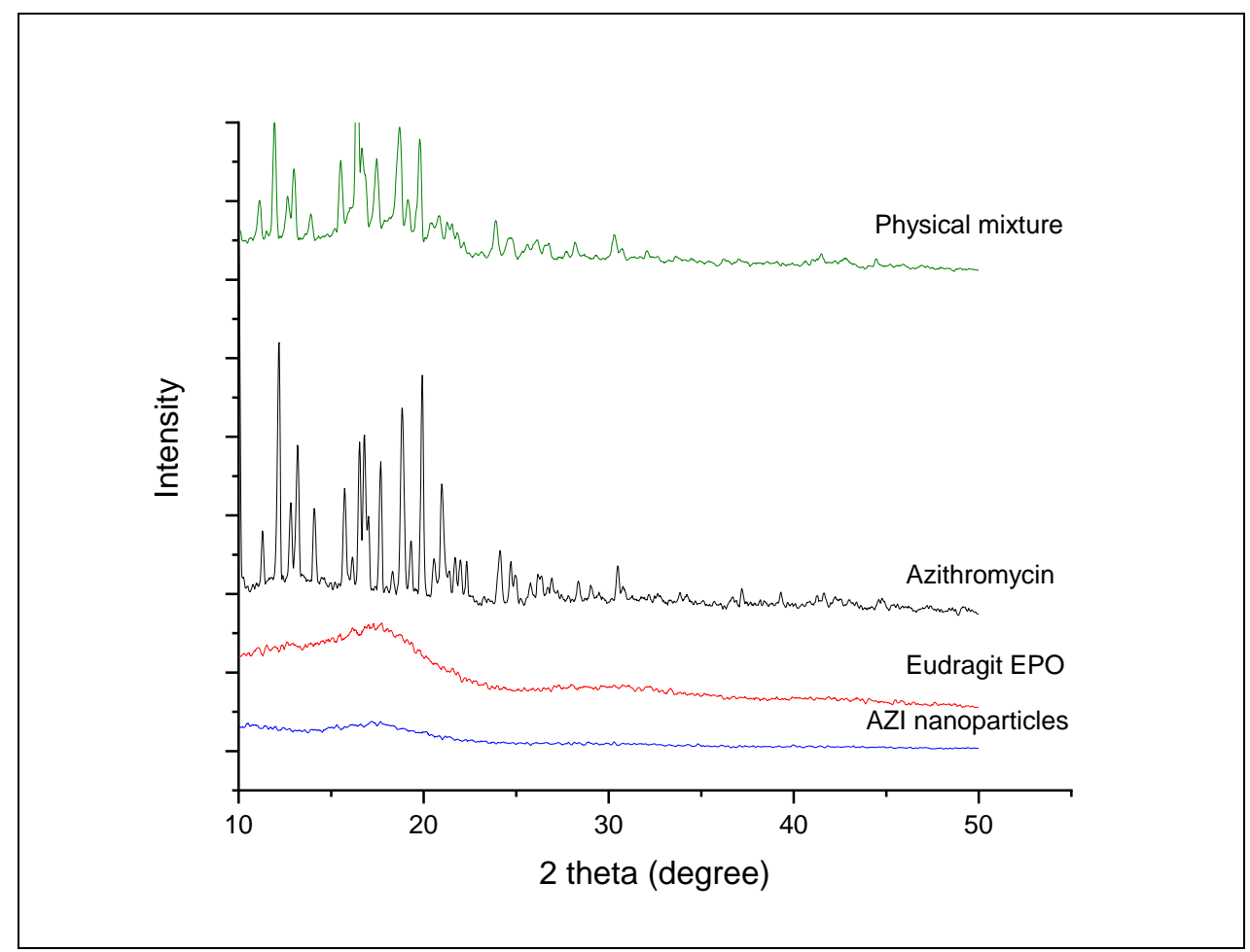

Figure 8. PXRD diffractograms azithromycin, Eudragit EPO, the physical mixture, and azithromycin nanoparticles. 


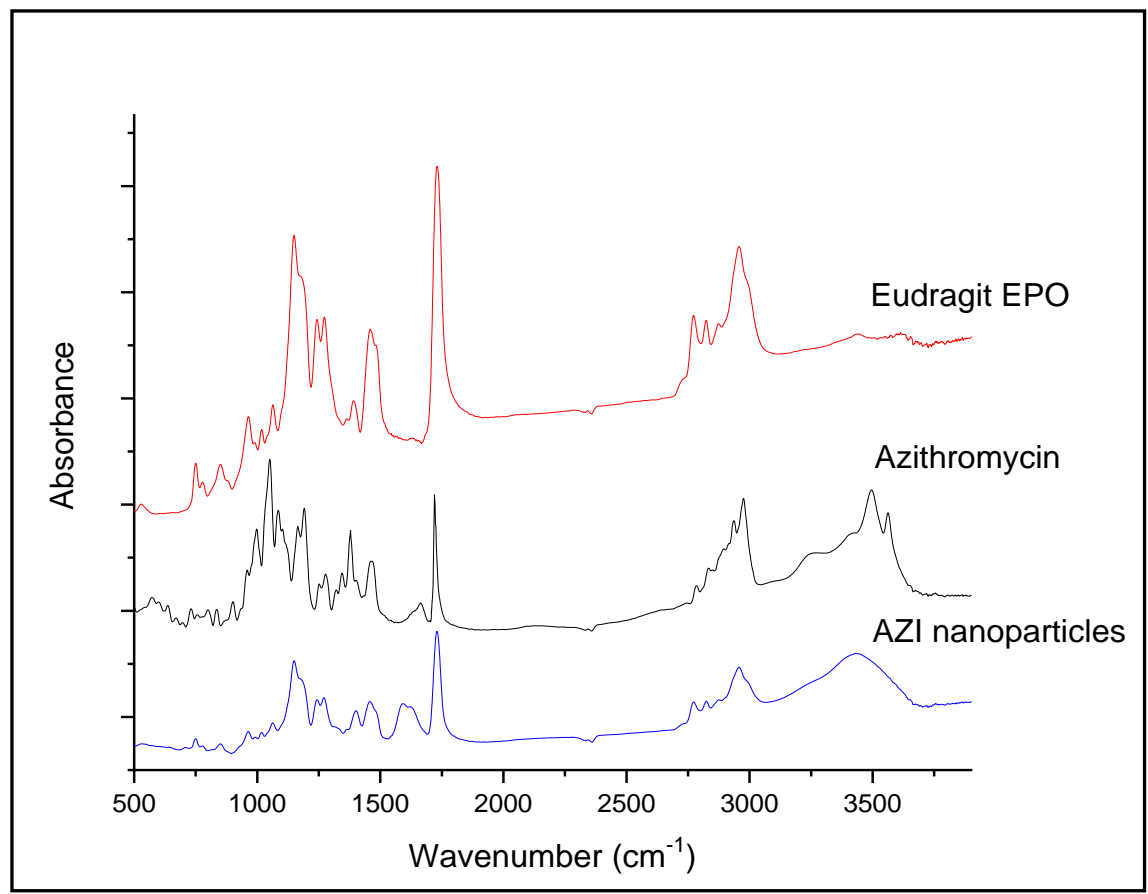

Figure 9. FT-IR spectra of azithromycin, Eudragit EPO, and azithromycin nanoparticles.

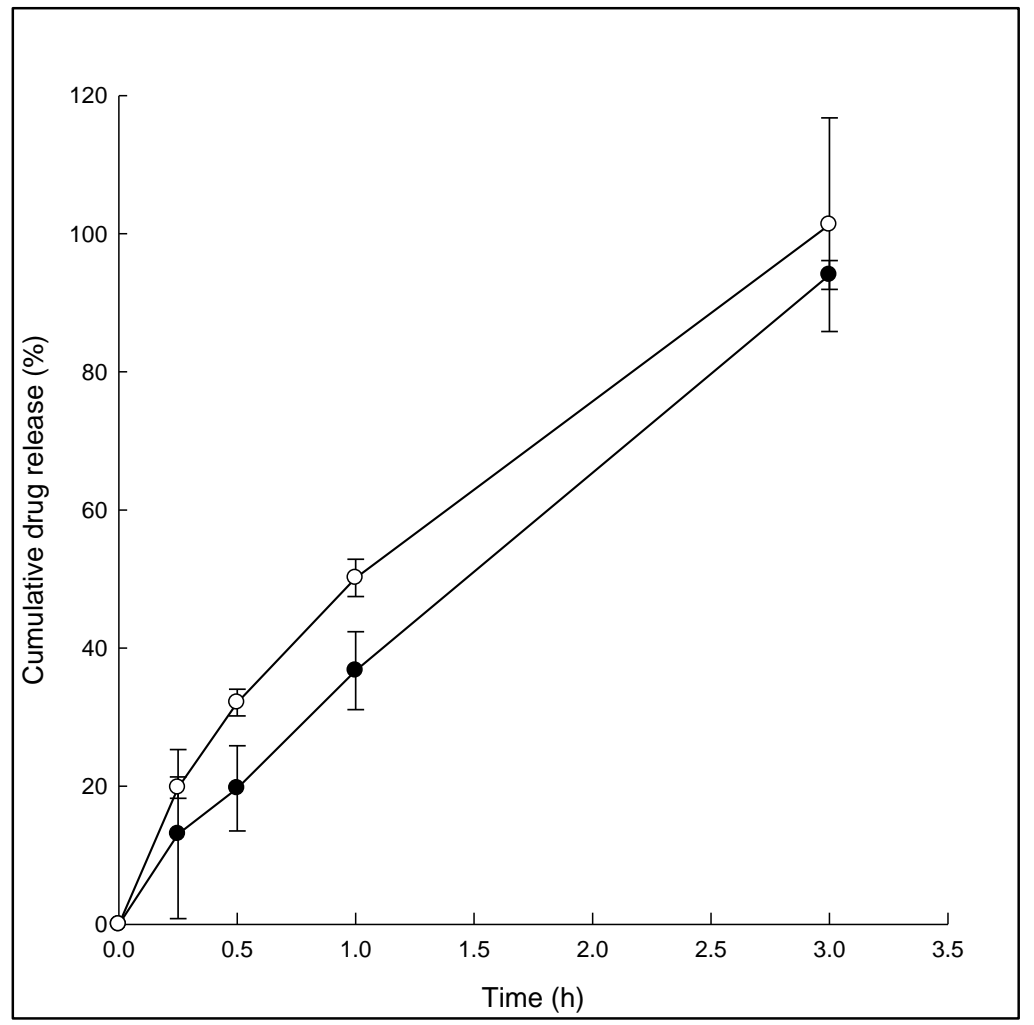

Figure 10. The drug release profile of $\circ)$ azithromycin nanoparticles and $\bullet$ ) azithromycin raw material $(\mathrm{n}=3$, Mean \pm S.D) .

Where $M_{t} / M_{\alpha}$ was the fraction released up to time $\mathrm{t}$; $\mathrm{k}$ was a constant incorporating structural and geometrical characteristics of dosage forms and $n$ was an exponent that described the release mechanism. The value of exponent coefficient ' $n$ ' was 1.17 , which indicated that the super case-II transport was the main drug transport mechanism. The drug transport was governed by relaxation of the polymer matrix and diffusivity of the drug dissolved ${ }^{35}$. Because Eudragit EPO, a low $\mathrm{Tg}$ polymer $\left(45^{\circ} \mathrm{C}\right)$, was completely soluble in dissolution medium ( $\mathrm{pH} 4.5)$, therefore it made the relaxation time almost instantaneous. Consequently, the driving force of drug release was the diffusivity of the drug dissolved. As the nanosize, the higher dissolution rate of AZI nanoparticles accelerated the drug diffusion rate out of dialysis membrane compared to the raw material. The 
fact that the value of a similar factor $\left(f_{2}=49\right)$ was under 50 indicated the nanoparticles had a higher drug release rate than the raw material.

\section{CONCLUSION}

In this study, the effect of two formulation variables on the characteristics of AZI nanoparticles was evaluated by the quality by design approach. The results showed that central composite face design was a proper way to determine the main effect, interaction, and quadratic effect on particle size, entrapment efficacy, and zeta potential of AZI-loaded Eudragit EPO nanoparticles. The results showed that the interaction of the ratio of AZI and volume of outer aqueous medium was significantly effective on drug entrapment efficacy.

\section{ACKNOWLEDGMENT}

For this paper, we don't have a scholarship or grant supporter.

\section{Conflict of interest}

The authors declare that they have no conflict of interest.

\section{Funding}

None to declare.

\section{Ethics approval}

None to declare.

\section{Article info:}

Received July 25, 2021

Received in revised form November 14, 2021

Accepted November 15, 2021

\section{REFERENCES}

1. Mora-Huertas CE, Fessi H, Elaissari A. Polymer-based nanocapsules for drug delivery. Int J Pharm. 2010;385(1-2):113-42.

2. Sarnes A, Kovalainen M, Hakkinen MR, Laaksonen T, Laru J, Kiesvaara J, et al. Nanocrystal-based per-oral itraconazole delivery: superior in vitro dissolution enhancement versus Sporanox ${ }^{\circledR}$ is not realized in in vivo drug absorption. J Control Release. 2014;180:109-16.

3. Cun D, Jensen DK, Maltesen MJ, Bunker M, Whiteside P, Scurr D, et al. High loading efficiency and sustained release of siRNA encapsulated in PLGA nanoparticles: quality by design optimization and characterization. Eur J Pharm Biopharm. 2011;77(1): 26-35.

4. Toti US, Guru BR, Hali M, McPharlin CM, Wykes SM, Panyam J, et al. Targeted delivery of antibiotics to intracellular chlamydial infections using PLGA nanoparticles. Biomaterials. 2011;32 (27):6606-13.

5. Webster DM, Sundaram P, Byrne ME. Injectable nanomaterials for drug delivery: carriers, targeting moieties, and therapeutics. Eur J Pharm Biopharm. 2013;84(1):1-20.

6. Bhavsar MD, Amiji MM. Development of novel biodegradable polymeric nanoparticles-in-microsphere formulation for local plasmid DNA delivery in the gastrointestinal tract. AAPS Pharm SciTech. 2008;9(1):288-94.
7. Vauthier C, Bouchemal K. Methods for the Preparation and Manufacture of Polymeric Nanoparticles. Pharmaceutical Research. 2009;26(5):1025-58

8. Hernández-Giottonini KY, Rodríguez-Córdova RJ, GutiérrezValenzuela CA, Peñuñuri-Miranda O, Zavala-Rivera P, GuerreroGermán P, et al. PLGA nanoparticle preparations by emulsification and nanoprecipitation techniques: effects of formulation parameters. RSC Advances. 2020;10(8):4218-31.

9. Mora-Huertas CE, Couenne F, Fessi H, Elaissari A. Electrokinetic properties of poly- $\varepsilon$-caprolactone-based nanoparticles prepared by nanoprecipitation and emulsification-diffusion methods: a comparative study. J Nanopart Res. 2012;14(6):1-15.

10. Mora-Huertas CE, Fessi H, Elaissari A. Influence of process and formulation parameters on the formation of submicron particles by solvent displacement and emulsification-diffusion methods: critical comparison. Adv Colloid Interface Sci. 2011;163(2): 90-122.

11. Sinha B, Müller RH, Möschwitzer JP. Bottom-up approaches for preparing drug nanocrystals: Formulations and factors affecting particle size. Int J Pharm. 2013;453(1):126-41.

12. Murakami H, Kobayashi M, Takeuchi H, Kawashima Y. Preparation of poly(dl-lactide-co-glycolide) nanoparticles by modified spontaneous emulsification solvent diffusion method. Int J Pharm. 1999;187(2):143-52.

13. Rahman Z, Zidan AS, Habib MJ, Khan MA. Understanding the quality of protein loaded PLGA nanoparticles variability by Plackett-Burman design. Int J Pharm. 2010;389(1-2):186-94.

14. Yerlikaya F, Ozgen A, Vural I, Guven O, Karaagaoglu E, Khan MA, et al. Development and evaluation of paclitaxel nanoparticles using a quality-by-design approach. J Pharm Sci. 2013;102(10): 3748-61.

15. Pharmaceutical Development Q8(R2). International Conference on Harmonisation of Technical Requirements for Registration of Pharmaceuticals for Human Use, ICH Harmonised Tripartite Guideline Current Step 4 Version. 2009.

16. Tung NT, Hung MV, Vo XM, Nguyen TH, Pham TM. Formulation optimization of orally disintegrating tablets containing solid dispersion of felodipine and hydroxypropyl methylcellulose using face-centered central composite design. J Pharm Investig. 2014;44(2):111-8.

17. Hou CD, Wang JX, Le Y, Zou HK, Zhao H. Preparation of azithromycin nanosuspensions by reactive precipitation method. Drug Dev Ind Pharm. 2012;38(7):848-54.

18. Stebbins ND, Ouimet MA, Uhrich KE. Antibiotic-containing polymers for localized, sustained drug delivery. Adv Drug Deliv Rev. 2014;78:77-87.

19. Pelgrift RY, Friedman AJ. Nanotechnology as a therapeutic tool to combat microbial resistance. Adv Drug Deliv Rev. 2013;65 (13-14):1803-15.

20. Sathigari SK, Radhakrishnan VK, Davis VA, Parsons DL, Babu RJ. Amorphous-state characterization of efavirenz--polymer hot-melt extrusion systems for dissolution enhancement. J Pharm Sci. 2012;101(9):3456-64.

21. Dos Santos J, da Silva GS. Eudragit(®): A Versatile Family of Polymers for Hot Melt Extrusion and 3D Printing Processes in Pharmaceutics. Pharmaceutics. 2021;13(9):1424.

22. Mora-Huertas CE, Fessi H, Elaissari A. Influence of process and formulation parameters on the formation of submicron particles by solvent displacement and emulsification-diffusion methods critical comparison. Adv Colloid Interface Sci. 2011;163(2): 90-122.

23. Zhang $Y$, Wang $X$, Lin $X$, Liu $X$, Tian B, Tang $X$. High azithromycin loading powders for inhalation and their in vivo evaluation in rats. Int J Pharm. 2010;395(1-2):205-14.

24. FDA, Center for Drug Evaluation and Research. Guidance for Industry: Dissolution Testing of Immediate Release Solid Oral Dosage Forms. Rockville, MD: U.S. Pharmacopeial Convention, Inc.; 1997.

25. Keum CG, Noh YW, Baek JS, Lim JH, Hwang CJ, Na YG, et al. 
Practical preparation procedures for docetaxel-loaded nanoparticles using polylactic acid-co-glycolic acid. Int J Nanomedicine. 2011;6:2225-34.

26. Sandhya M, Ramasamy D, Sudhakar K, Kadirgama K, Harun WSW. Ultrasonication an intensifying tool for preparation of stable nanofluids and study the time influence on distinct properties of graphene nanofluids-A systematic overview. Ultrason Sonochem. 2021;73:105479.

27. Teychené S, Rodríguez-Ruiz I, Ramamoorthy RK. Reactive crystallization: From mixing to control of kinetics by additives. Curr Opin Colloid Interface Sci. 2020;46:1-19.

28. Pineda-Reyes AM, Hernández Delgado M, Zambrano-Zaragoza MdIL, Leyva-Gómez G, Mendoza-Muñoz N, Quintanar-Guerrero D. Implementation of the emulsification-diffusion method by solvent displacement for polystyrene nanoparticles prepared from recycled material. RSC Advances. 2021;11(4):2226-34.

29. Rhee YS, Chang SY, Park CW, Chi SC, Park ES. Optimization of ibuprofen gel formulations using experimental design technique for enhanced transdermal penetration. Int J Pharm. 2008;364(1): 14-20.

30. Zhao X, Liu Y, Song J, Yong H, Li L, Shen Y, et al. Cationic lipids percentage and processing temperature are critical in designing siRNA lipid nanoparticles. J Drug Target. 2012;20(3):281-9.

31. Liandong H, Jianbin P, Ci L, Hongxin X, Liangzhao L. Preparation, characterization and taste-masking properties of microspheres containing azithromycin. J Pharm Pharmacol. 2009;61 (12):1631-5.

32. Li X, Peng H, Tian B, Gou J, Yao Q, Tao X, et al. Preparation and characterization of azithromycin--Aerosil 200 solid dispersions with enhanced physical stability. Int J Pharm. 2015;486(12):175-84.

33. Zhang D, Tan T, Gao L, Zhao W, Wang P. Preparation of azithromycin nanosuspensions by high pressure homogenization and its physicochemical characteristics studies. Drug Dev Ind Pharm. 2007;33(5):569-75.

34. Tung NT, Tran CS, Nguyen TL, Hoang T, Trinh TD, Nguyen TN. Formulation and biopharmaceutical evaluation of bitter taste masking microparticles containing azithromycin loaded in dispersible tablets. Eur J Pharm Biopharm. 2018;126:187-200.

35. Edwards DA, Cohen DS. The effect of a changing diffusion coefficient in super-Case II polymer-penetrant systems. IMA J Appl Math. 1995;55(1):49-66. 